\title{
Recent results from CMS
}

\author{
Milos Dordevic ${ }^{1}$ on behalf of the CMS Collaboration * \\ ${ }^{1}$ Vinca Institute of Nuclear Sciences, PO Box 522, 11001 Belgrade, Serbia
}

\begin{abstract}
The highlights of the most recent CMS results with $13 \mathrm{TeV}$ data will be presented in this overview. The Standard Model precision measurements, including the top quark production, will be shown first. This will be followed by the presentation of Higgs boson studies with the early $13 \mathrm{TeV}$ data. Then the focus will shift to searches for physics beyond the Standard Model, including the searches for several Supersymmetric scenarios, using different analysis techniques. The talk will conclude with searches for the exotic resonances, with an emphasis on studies of the high-mass diphoton production.
\end{abstract}

\section{Introduction}

The LHC Run I was very successful for the CMS Collaboration, with the major event being the discovery of the Higgs boson. A large number of studies of the known processes and searches for new phenomena was conducted, all of them confirming the prediction of the Standard Model (SM) with the unprecedented precision. The first studies of $13 \mathrm{TeV}$ data recorded by the CMS experiment in the year of 2015 will be presented in this overview.

\section{Standard Model results at $13 \mathrm{TeV}$}

\subsection{Studies of two-particle correlations}

The two-particle angular correlations for charged particles are studied at $13 \mathrm{TeV}$ [1]. The presence of the ridge-like structure for long range $(|\eta|>2.0)$ near-side $\Delta \phi \approx 0$ pairs is confirmed, as shown in Fig. 1. The magnitude of the correlation is maximal in the range $1.0<p_{T}<2.0 \mathrm{GeV} / \mathrm{c}$, with an approximately linear increase with the charge particle multiplicity, similar to the one measured at the $7 \mathrm{TeV}$ [2], but now extended to much higher multiplicity values. Strong collision system size dependence is observed when comparing data from $\mathrm{pp}, \mathrm{pPb}$ and $\mathrm{PbPb}$ collisions.

\section{$2.2 \mathrm{~W}$ and $\mathrm{Z}$ boson production}

Total inclusive and fiducial $\mathrm{W}$ and $\mathrm{Z}$ boson cross sections at $13 \mathrm{TeV}$ are measured in electron and muon final states [3]. The total inclusive cross sections times branching fractions are $\sigma\left(p p \rightarrow W^{+} X \times\right.$ $B\left(W^{+} \rightarrow l^{+} v\right)=11370 \pm 50($ stat $) \pm 230($ syst $) \pm 550($ lumi $) p b, \sigma\left(p p \rightarrow W^{-} X \times B\left(W^{-} \rightarrow l^{-} v\right)=\right.$ $8580 \pm 50($ stat $) \pm 160($ syst $) \pm 410($ lumi $) p b$ and $\sigma\left(p p \rightarrow Z X \times B\left(Z \rightarrow l^{+} l^{-}\right)=1910 \pm 10(\right.$ stat $) \pm$

^e-mail: milos.dordevic@cern.ch 
40 (syst) \pm 90 (lumi) pb for the dilepton mass range of 60 to $120 \mathrm{GeV}$. The measured ratio of the $\mathrm{W}$ and $\mathrm{Z}$ boson cross section in the electron and muon channels, that is useful also as an additional check of the lepton universality, is shown in Fig. 2. The measurement is found to be in a good agreement with the SM prediction.

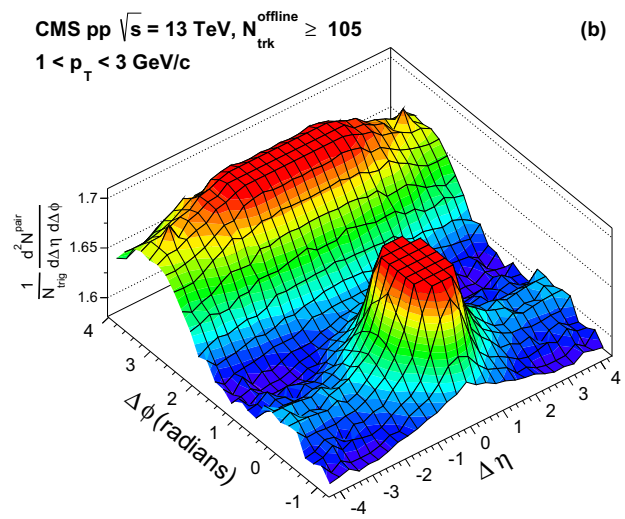

Figure 1. The two-particle correlation function for pairs of charge particles both in range 1 $<p_{T}<3 \mathrm{GeV} / \mathrm{c}$, showing the "rigde"-like structure.

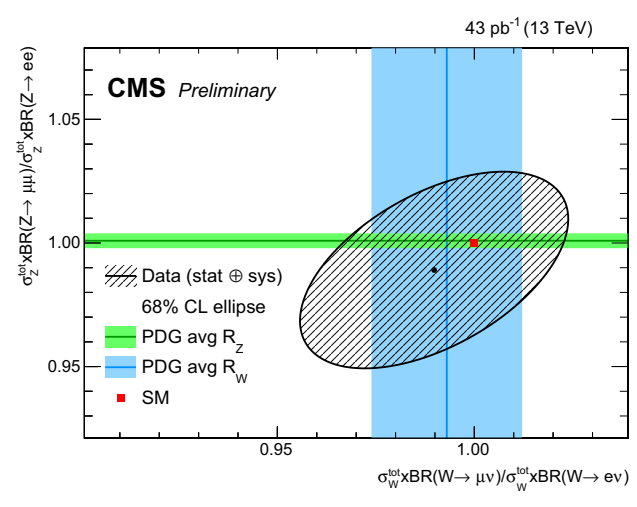

Figure 2. Ratios of $\mathrm{W}$ and $\mathrm{Z}$ inclusive cross sections in the electron and muon channels, as test of lepton universality and the SM expectation.

\subsection{Inclusive jet production}

Double-differential inclusive jet cross section, as a function of jet transverse momentum and rapidity, is measured for two jet size parameters $\mathrm{R}=0.7$ and $\mathrm{R}=0.4$ and jets with $p_{T}$ up to $2 \mathrm{TeV}$ and $|y|=$ 4.7 [4] and the results are presented in Fig. 3. The new energy of $13 \mathrm{TeV}$ provides an access to kinematic region of momentum fraction $\mathrm{x}$ where the parton density is large. When comparing the data to predictions at NLO accuracy in PQCD, a better agreement is observed for the larger jet size. The NLO prediction matched to parton shower from POWHEG+PYTHIA8 [5, 6] performs equally well for the both jet cone size parameters. The LO predictions of PYTHIA8 and HERWIG++ [7] have significant discrepancies with respect to data, thus stressing the importance of the higher order corrections.

\subsection{Single top production}

The top quark mass was measured in single top events [8], where the $\mathrm{W}$ boson decays to muon and a neutrino. Specific event topology and kinematics, featuring the central production of a light jet and an asymmetry of the initial state, was exploited to suppress the ttbar background. The measured value of $m_{t}=172.60 \pm 0.77(\text { stat })_{-0.93}^{+0.97}($ syst $) \mathrm{GeV}$, extracted from a fit to the top mass distribution (Fig. 4), is in a good agreement with the world average of $m_{t}=173.34 \pm 0.76 \mathrm{GeV}$. The t-channel single top cross section at $13 \mathrm{TeV}$ is measured as well [9]. Kinematic variables are combined into a multivariate discriminator that was fit to obtain the inclusive cross section of $\sigma_{t-c h}=227.8 \pm$ 9.1 (stat. $) \pm 14.0(\text { exp. })_{-27.7}^{+28.7}($ theo. $) \pm 6.2$ (lumi. $) p b=227.8_{-33.0}^{+33.7} p b$, found to be in a good agreement with the SM expectation. 

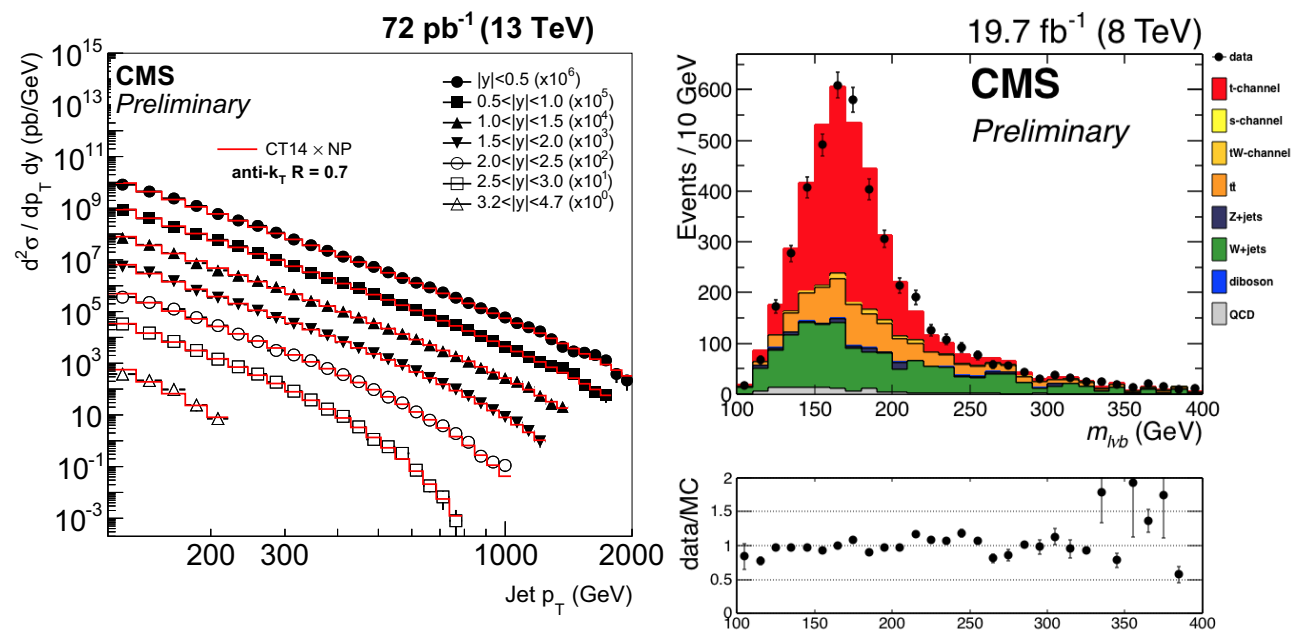

Figure 3. Double-differential inclusive jet cross section, shown as a function of the jet transverse momentum.

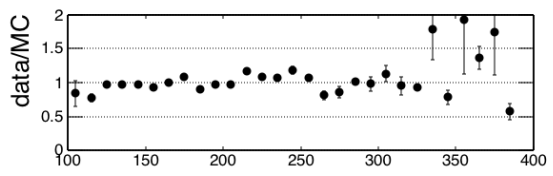

Figure 4. The top quark mass after the final selection including also the charge and pseudorapidity cuts.

\section{Higgs studies at the $13 \mathrm{TeV}$}

\section{1 ttH production}

A search for SM Higgs boson in association with a top quark pair at $13 \mathrm{TeV}$ is performed. The analysis targets the bbar, $\gamma \gamma$ and multilepton decays of the Higgs boson. The multilepton analysis selects final states with two same-sign leptons or more than three leptons produced in association with $b$ jets [10]. The signal extraction is based on the two-dimensional multivariate discriminator trained against each of the ttbar and $\mathrm{ttV}$ backgrounds, as shown in Fig. 5. The signal strenght is measured to be the $0.6_{-1.1}^{+1.4}$ times the SM expectation and the observed (expected) 95\% CL limits are 3.3 (2.6) times the SM expectation. For the Higgs boson decays to a pair of $\mathrm{b}$ quarks, the lepton plus jets and dileptonic decays of the ttbar system are considered [11]. A combination of the matrix element method and multivariate approach is performed. A combined fit of the multivariate discriminant templates to data yielded with the signal strength of $-2.0_{-1.8}^{+1.8}$ and the observed (expected) 95\% CL limits are 2.6 (3.6) times the SM expectation. The Higgs to diphotons analysis, although statistically limited at this point, was performed with the inclusion of the two categories with leptonic and hadronic tags of the ttbar pair [12]. The signal strength for the $\mathrm{ttH}$ production in this channel is $-3.8_{-3.6}^{+4.5}$, with the two tags corresponding to the $\mathrm{ttH}$ process combined statistically.

\subsection{Higgs decays to bosons}

The diphoton channel remains one of the best to test Higgs properties. It was performed in the $\mathrm{gg} \rightarrow \mathrm{H}$, $\mathrm{VBF}$ and $\mathrm{ttH}$ categories [12]. Small deficit that is observed is driven by the untagged categories $(\mathrm{gg} \rightarrow \mathrm{H})$. The observed (expected) significance at $125.09 \mathrm{GeV}$ is $1.7(2.7) \sigma$ and the signal strength of $0.69_{-0.42}^{+0.47}$ is consistent with the SM expectation, as shown in Fig. 7. The $\mathrm{H} \rightarrow \mathrm{ZZ} \rightarrow 41$ analysis, based on the matrix element kinematic discriminant between signal and background, is performed [13]. The distribution of the four lepton invariant mass is shown in Fig. 8. The observed(expected) significance 


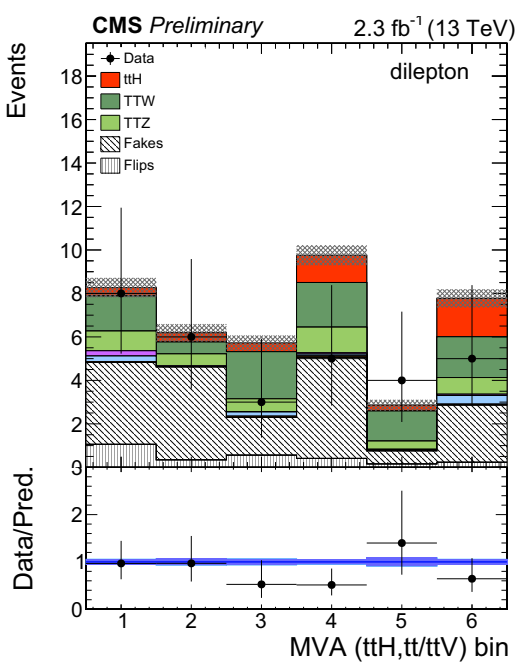

Figure 5. BDT kinematic discriminant for signal extraction in two same-sign lepton category.

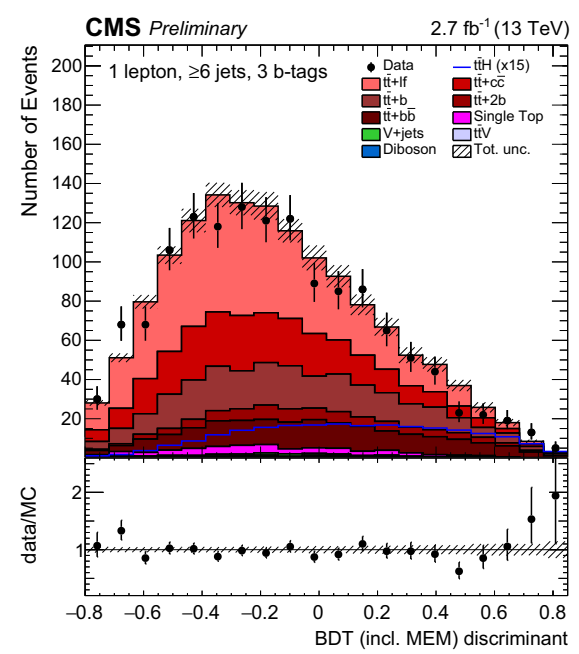

Figure 6. Final discriminator shape of the BDT, including also the MEM discriminator in the training.

at $125.09 \mathrm{GeV}$ of $2.5(3.4) \sigma$ and the signal strength of $0.82_{-0.43}^{+0.57}$ are consistent with the SM within the uncertainties.

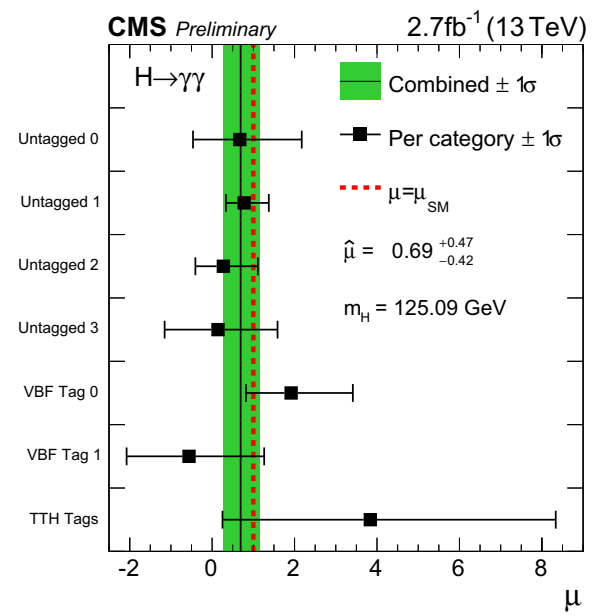

Figure 7. Signal strength modifier for each category in $\mathrm{H} \rightarrow \gamma \gamma$ channel, compared to the overall signal strength and to the SM expectation.

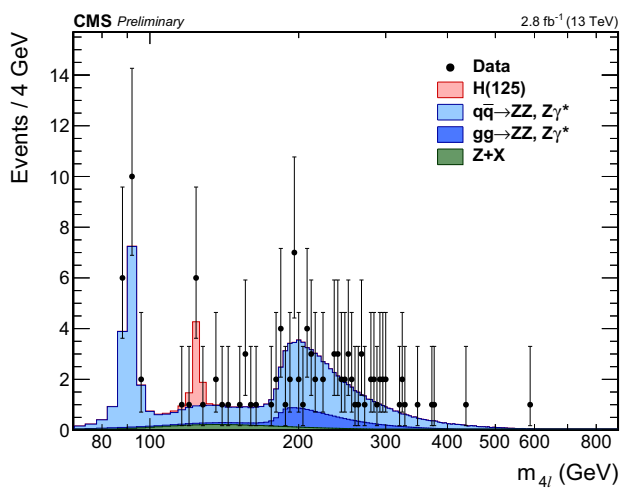

Figure 8. The four lepton invariant mass distribution $m_{4 l}$, shown in the full studied mass range. 


\section{Supersymmetry searches at the $13 \mathrm{TeV}$}

\subsection{SUSY with zero leptons}

The searches for gluino pair production with the LSP and zero leptons in the final state are performed [14]. The results of the analysis using the HT variables, as shown in Fig. 9, provides some of the world leading limits on T1bbbb, T1tttt and T1qqqq of 1600, 1530 and $1440 \mathrm{GeV}$. Similar search was performed using the stransverse mass variable $M_{T 2}$, which is a measure of the transverse momentum imbalance in an event [15]. No excess above the SM background is observed and gluino(neutralino) mass was probed up to $1725(1100) \mathrm{GeV}$, as shown in Fig. 10, extending the Run 1 sensitivity by more than $300 \mathrm{GeV}[16]$.

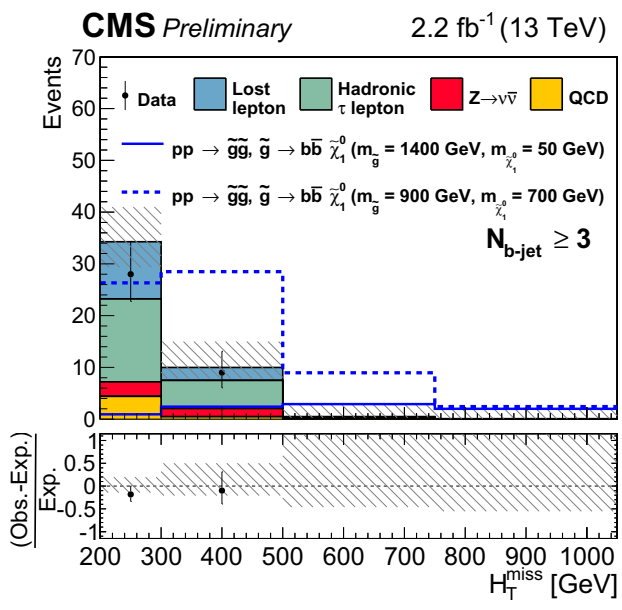

Figure 9. Observed number of events and SM background predictions for one of the search regions sensitive to the T1bbbb scenarios.

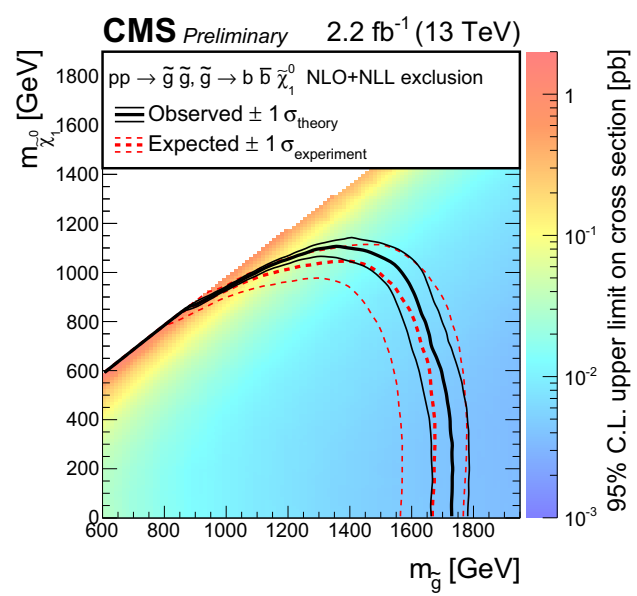

Figure 10. Exclusion limit at 95\% CL for gluino mediated bottom-squark production.

\subsection{SUSY with one lepton}

The zero lepton SUSY search using the Razor variables is extended with an additional one lepton category [17]. The events are further categorised by the number of b-tagged jets in the final state. For the $100 \mathrm{GeV}$ neutralino mass and gluino decaying to bb pairs, the limits are set to $1650 \mathrm{GeV}$, while for the decays to top(light) quarks this particular SUSY scenario is excluded up to 1600 (1350) GeV, as shown in Fig. 11. The search for SUSY in final state with one lepton, one b-tagged jet and large missing transverse energy (MET) was performed using the sum of masses of large radius jets [18]. A key feature of this analysis is that the dominant background ttbar is nearly uncorrelated with the transverse mass of the lepton and MET. The distribution of the $M_{J}$ variable is shown in Fig. 11 . Within the T1tttt scenario the gluino mass is excluded up to $1575 \mathrm{GeV}$.

\section{Exotica searches at the $13 \mathrm{TeV}$}

\subsection{Search for black holes}

The search for black holes is performed [19] in the framework of ADD model with n-extra dimensions [20-22]. The semiclassical and quantum black holes are considered. The SM background, dominated 


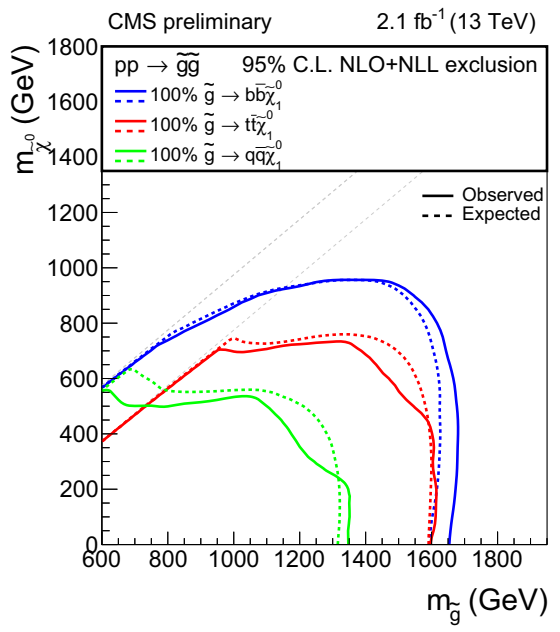

Figure 11. Summary of the exclusion contours at $95 \% \mathrm{CL}$, with gluino decays at $100 \%$ branching fraction to LSP and bb, tt and qq pairs.

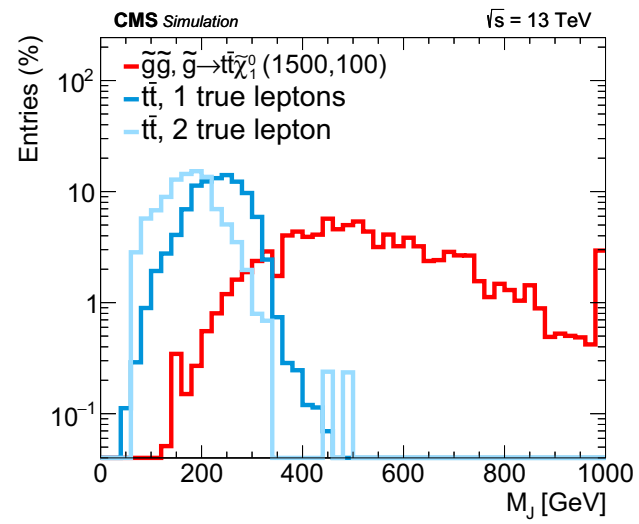

Figure 12. Distribution of $M_{J}$ from simulated events with a small amount of initial state radiation (ISR).

by the QCD multijet events, is estimated from data. The distribution of the discriminating variable $S_{T}$, representing a scalar sum of the transverse energies of all energetic objects in the event, is shown in Fig. 13. No significant excess has been observed and the 95\% CL limits in the semiclassical(quantum) black hole interpretation of 8.7(8.0) TeV are established. These limits are surpassing the $8 \mathrm{TeV}$ results of $5.5-6.0 \mathrm{TeV}[23]$.

\subsection{Search for dark matter}

The search for dark matter in the final state with large MET and at least one jet with high transverse momentum is performed [24]. The dark matter is considered to be produced in pairs, via the decay of a vector mediator, and the signal is extracted from a fit to the observed MET distribution in data. The mediator mass up to $1.3 \mathrm{TeV}$ is excluded at $90 \% \mathrm{CL}$, as it is shown in Fig. 14 where also the results from the other, non-collider experiments, are overlaid together with this measurement, for the comparison.

\subsection{Search for diphoton resonances}

The search for diphoton resonances, under the 2HDM (spin 0) or ADD/RS (spin 2) hypothesis, is performed [25]. The high diphoton mass region between the $500 \mathrm{GeV}$ and $4.5 \mathrm{TeV}$ is explored, with the relative width up to $5.6 \times 10^{-2}$. The $13 \mathrm{TeV}$ results are combined with similar searches at the 8 $\mathrm{TeV}[26,27]$. The largest excess is observed at $750 \mathrm{GeV}$ and resonance width of $1.4 \times 10^{-4}$, with the local p-value of $3.4 \sigma$, reduced to $1.6 \sigma$ when taking into account of searching for the several signal hypotheses. The corresponding diphoton mass distribution and p-value are shown in Fig. 15 and Fig. 16 , respectively. 


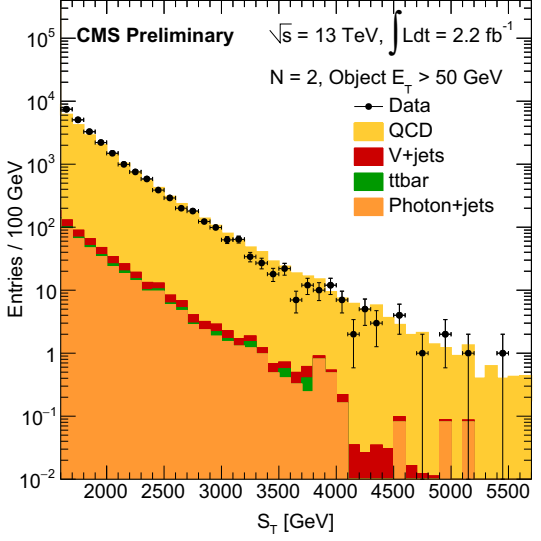

Figure 13. The $S_{T}$ variable distribution, with the main contribution from QCD multijet background.

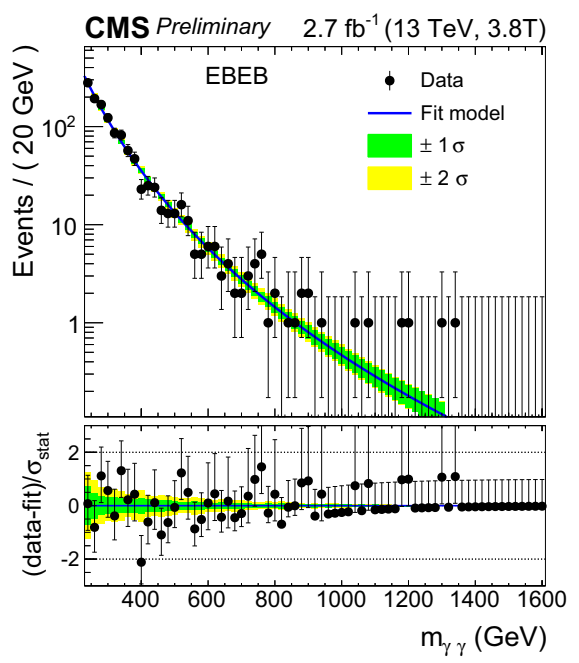

Figure 15. The observed invariant mass spectra for the EBEB channel and using the 3.8T dataset.

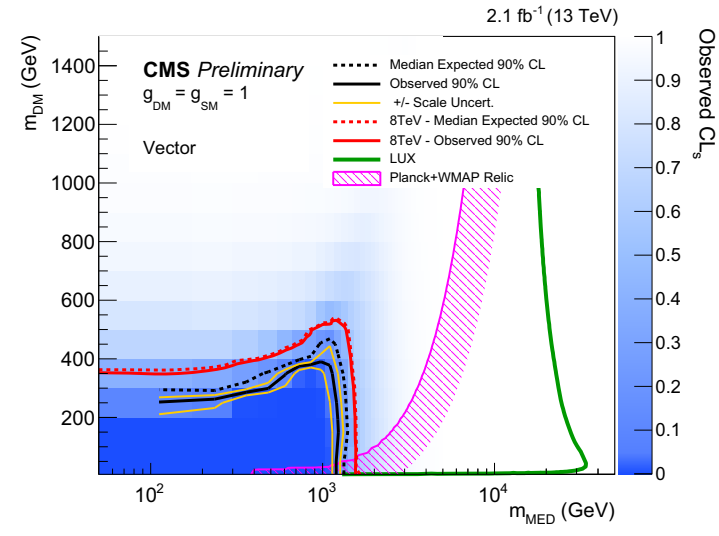

Figure 14. The dark matter exclusion contours in the $m_{\text {med }}-m_{D M}$ plane assuming a vector-type interaction.

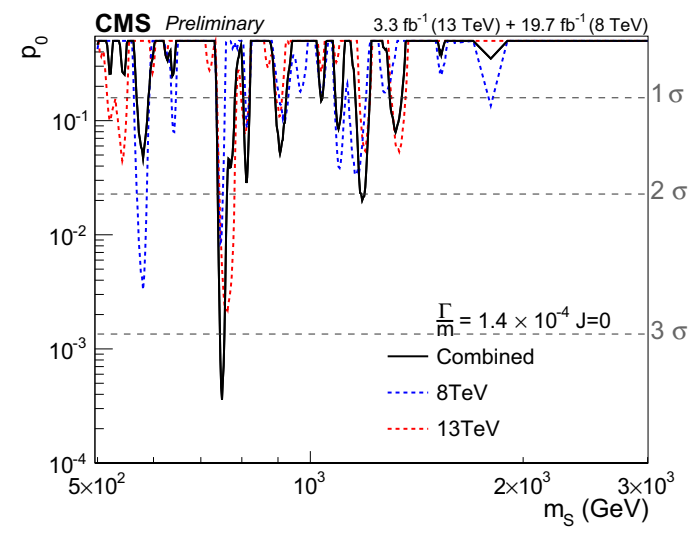

Figure 16. The observed background only pvalues for spin 0 and the most narrow width hypothesis, obtained from the combination of the 8 and $13 \mathrm{TeV}$ results.

\section{Conclusion}

The 2015 has marked a start of CMS taking the $13 \mathrm{TeV}$ data. It was the first operation under the $25 \mathrm{~ns}$ conditions and physics objects are well commissioned. The SM results are generally in an agreement with the prediction, many new physics results have an improved sensitivity and an excess in the diphoton invariant mass is observed at $750 \mathrm{GeV}$, that requires more data for a precise scrutiny of its existence. 


\section{References}

[1] The CMS Collaboration, Phys. Rev. Lett. 116, 172302, (2016)

[2] The CMS Collaboration, JHEP 09 091, (2016)

[3] The CMS Collaboration, CMS-PAS-SMP-15-004, (2016)

[4] The CMS Collaboration, CMS-PAS-SMP-15-007, (2016)

[5] T. Sjostrand, S. Mrenna and P. Skands, JHEP 05 026, (2006)

[6] T. Sjostrand, S. Mrenna and P. Skands, Comput. Phys. Comm. 178 852, (2008)

[7] G. Corcella et al., JHEP 0101 010, (2001)

[8] The CMS Collaboration, CMS-PAS-TOP-15-001, (2016)

[9] The CMS Collaboration, CMS-PAS-TOP-16-003, (2016)

[10] The CMS Collaboration, CMS-PAS-HIG-15-008 (2016)

[11] The CMS Collaboration, CMS-PAS-HIG-16-004, (2016)

[12] The CMS Collaboration, CMS-PAS-HIG-15-005, (2016)

[13] The CMS Collaboration, CMS-PAS-HIG-15-004, (2016)

[14] The CMS Collaboration, CMS-PAS-SUS-15-002 (2016)

[15] The CMS Collaboration, CMS-PAS-SUS-15-003, (2016)

[16] The CMS Collaboration, JHEP 05 078, (2015)

[17] The CMS Collaboration, CMS-PAS-SUS-15-004, (2016)

[18] The CMS Collaboration, CMS-PAS-SUS-15-007, (2016)

[19] The CMS Collaboration, CMS-PAS-EXO-15-007, (2016)

[20] N. Arkani-Hamed, S. Dimopoulos, G.R. Dvali, Phys. Lett. B 429 264, (1988)

[21] N. Arkani-Hamed, S. Dimopoulos, G.R. Dvali, Phys. Lett. B 436 257, (1988)

[22] N. Arkani-Hamed, S. Dimopoulos, G.R. Dvali, Phys. Rev. D 59 086004, (1999)

[23] G. Landsberg, Fundam. Theor. Phys., 178267 (2015)

[24] The CMS Collaboration, CMS-PAS-EXO-15-003, (2016)

[25] The CMS Collaboration, CMS-PAS-EXO-16-018, (2016)

[26] The CMS Collaboration, Phys. Lett 750 494-419, (2016)

[27] The CMS Collaboration, CMS-PAS-EXO-12-045, (2016) 